\title{
Crimen transnacional organizado y utilitarismo sociológico: evidencia desde el tráfico de migrantes en Colombia
}

\author{
Farid Badrán Robayo* \\ Mauricio Palma Gutiérrez
}

\section{RESUMEN}

El utilitarismo sociológico -o el compendio de motivaciones no económicas, y no estrictamente racionales que se internalizan en agentes sociales y por las cuales dichos actores tienden a reproducir lógicas criminales- se manifiesta de diferentes formas en el crimen transnacional. Varios elementos, por fuera del análisis tradicional fundado en el racionalismo económico, pueden ser expuestos a través de esta perspectiva. Este texto propone una agenda de análisis con cinco puntos concretos por observar respecto a la generación de motivaciones sociológicas frente al crimen transnacional: el usufructo y la proyección de la autoridad; la necesidad de suscribirse a estructuras de protección en zonas grises de presencia estatal; el interés por generar, alimentar, preservar o contribuir a la generación identitaria; la necesidad de crear mecanismos paralelos de regulación social; la necesidad de financiar causas consideradas moralmente superiores. Su aplicación frente a la evolución del tráfico de migrantes en Colombia se convierte en una primera oportunidad para comenzar a pensar en el tema, de cara al entendimiento de otros fenómenos similares.

* MSc, Estudios Latinoamericanos, Universidad Paris III Sorbona Nueva. Profesor de Relaciones Internacionales, Universidad Javeriana, Bogotá (Colombia) [fbadran@dnp.gov.co].

* MSc, Global History, London School of Economics. Profesor de Relaciones Internacionales, Universidad del Rosario, Bogotá (Colombia) [mauricio.palma@urosario.edu.co].

Recibido: 11 de julio de 2016/ Modificado: 20 de agosto de 2016/ Aceptado: 12 de septiembre de 2016

Para citar este artículo

Badrán Robayo, F. y Palma Gutiérrez, M. (2017). Crimen transnacional organizado y utilitarismo sociológico: evidencia desde el tráfico de personas en Colombia. OAsıs, 25, 77-94.

DOI: https://doi.org/10.18601/16577558.n25.05 
Palabras clave: utilitarismo sociológico, crimen transnacional, tráfico de migrantes, Colombia.

\section{Transnational organized crime and sociological utilitarianism: evidence from the traffic of migrants in Colombia}

\section{ABSTRACT}

Sociological utilitarianism, or the compendium of non-economic and not strictly rational motivations that are internalized in social agents due to which actors tend to reproduce criminal logic, is manifested through different forms of transnational crime. Several elements can be exposed though this perspective, outside of the traditional anaylsis based on economic rationalism. This text proposes an analysis agenda with five concrete points to observe in regards to the generation of sociological motivations facing transnational crime. These are: the usufruct and projection of authority; the need to subscribe to protection structures in grey areas of state presence; the interest to generate, nourish, preserve, or contribute to the generation of identity; the need to create parallel mechanisms of social regulation; and the need to finance causes considered to be morally superior. Its application to the evolution of the traffic of migrants in Colombia becomes a first opportunity to start thinking about the issue, along with the understanding of similar phenomena.

Key words: Sociological utilitarianism, transnational crime, migrant trafficking, Colombia.

\section{UTILITARISMO SOCIOLÓGICO DEL CRIMEN TRANSNACIONAL ORGANIZADO}

El crimen transnacional organizado -o el compendio de actividades ejecutadas para obtener un beneficio material a través de la comisión de un delito de manera grupal, y cuyo desarrollo traspasa las fronteras físicas e institucionales de un único Estado- se reproduce y transforma masivamente en diferentes latitudes. Esto deja entrever la obsolescencia e ineficacia de las medidas jurídicas, políticas y socioeconómicas que se han utilizado hasta ahora para neutralizar el fenómeno.

El crimen transnacional persiste en forma de modernas y gigantescas macrorredes que controlan todo tipo de flujos ilegales a través de las fronteras nacionales, en diferentes puntos de los cinco continentes. El desgobierno de estos espacios y la continua rentabilidad de las actividades que sobre estos se extienden propician la continuidad de un fenómeno que está aún lejos de ser controlado (Berdal y Serrano, 2002; Rabasa et al., 2007; Paoli, 2014).

Además, el alcance de este fenómeno sobrepasa el del puro espacio físico entre Estados. Los espacios sociales en los que se desenvuelven los actores criminales, las actividades que generan réditos económicos en medio de esta 
dinámica y los efectos que se generan a largo plazo, reflejan la dinámica transnacional de las relaciones sociales hoy. Vertovec (1999, p. 1) asegura que este transnacionalismo puede verse en como un "sistema de vínculos, interacciones, intercambio y movilidades" -como las redes que se mencionaron-, que sobrepasan las fronteras físicas de los Estados y que, "pese a las grandes distancias y las fronteras políticas", se perfila como un "espacio de interacción común” cuya extensión es global.

Pensando en crimen transnacional, son pocos los incentivos para que los protagonistas de estas redes dejen de lado su negocio. Desde hace años, la prisión dejó de ser un elemento disuasivo de las actividades desviadas de la norma social (Lyman y Potter, 2007). Por el contrario, estas se han convertido de manera progresiva en el centro geopolítico urbano de destrucción del Estado y en una oficina de la criminalidad organizada y del terrorismo fuera y dentro de sus muros (Vidino y Brandon, 2012).

Además, la alta probabilidad que tienen los agentes ilegales de morir, connatural al hecho de plegarse hacia lo criminal, tampoco resulta ser, per se, un elemento inhibitorio de la criminalidad. Este es incluso un efecto altamente valorado en el desarrollo de amenazas como el terrorismo asociado al fundamentalismo islámico, por ejemplo (Schneiders, 2014; Rahimi y Graumans, 2015). En el caso del crimen transnacional organizado, la muerte es uno más de los efectos colaterales asociados a su actividad y así lo han asumido sus practicantes, quienes naturalmente buscan preservar su vida, pero siguen desarrollando al mismo tiempo actividades ilícitas que pueden acarrear el fin de la misma. Dicho de otro modo, los criminales organizados tienen conciencia de la muerte como otro factor más en el obligado cálculo costo-beneficio derivado de sus ejercicios.

En función de lo anterior, es posible sustentar la tesis de que el crimen transnacional organizado es, en esencia, una actividad racionalmente elegible y ejecutorialmente viable por cuanto los elementos que se creían disuasivos no tienen el peso suficiente para reconducir las decisiones de los actores sociales hacia la legalidad (Badrán, 2014).

Concentrados en el constreñimiento del individuo, los sistemas judiciales y sus instrumentos penales parecen haber dejado de lado el principal motor que hace al crimen organizado posible, reproducible y rentable: la capacidad de adquirir bienes y servicios con dinero obtenido ilegalmente; es decir, a través del lavado de activos y la corrupción (Shelley, 1995). Ello supondría, por supuesto, una vigilancia más estrecha $y$, acaso, una suerte de securitización del sistema financiero y empresarial. En esto surgirían actores que usualmente no se agregan a la fórmula política de la construcción de amenazas a la seguridad por cuanto esto constituiría un comportamiento antijerárquico que no cabe en sistemas sociales y políticos construidos sobre la lógica de la autoridad, la dominación y el poder.

Justamente por esa capacidad reproductiva, y por el hecho de ser un comportamiento oportunista de adaptación al sistema (Rivelois, 2004), el crimen transnacional organizado empieza a denotar, en no pocos casos, una serie de epifenómenos derivados de su existencia en los sistemas sociales. En ese sentido, 
el utilitarismo del crimen organizado empieza a trascender la esfera económica en la medida en que así lo hacen también sus prácticas y representaciones. Una de las más notables tendencias del fenómeno da cuenta de niveles crecientes de regulación social alterna a la de la institucionalidad estatal.

En función de lo anterior, sería posible empezar a observar una suerte de utilitarismo sociológico derivado del crimen organizado que va más allá de las obvias consideraciones económicas y que, a su vez, trasciende las reflexiones deterministas que guiaron el debate durante décadas.

De tal forma, el utilitarismo sociológico del crimen organizado podría considerarse como el compendio de motivaciones no económicas $-\mathrm{y}$ no estrictamente racionales- que se internalizan en agentes sociales y por las cuales dichos actores tienden a reproducir el fenómeno. El alcance de esas motivaciones es amplio y su naturaleza depende del interés de quien las albergue, toda vez que no es lo mismo la necesidad que tenga un líder narcotraficante de establecer un cierto control social, que el interés de un facilitador por ganar respeto en una estructura criminal.

Joseph Albini y Jeffrey McIllwain (2012) hacen referencia de manera tangencial al aspecto sociológico inherente al crimen organizado al afirmar que los grupos de crimen organizado buscan, además de poder económico, influir y determinar ciertos cambios sociales en las esferas públicas y privadas.

En otras palabras, el utilitarismo sociológico del crimen organizado se desglosa y se adapta a la naturaleza de los actores que lo ejecutan y a las estructuras en las que ellos se desenvuelven. Las razones que componen este utilitarismo son múltiples. No obstante, es posible agruparlas en cinco grandes intereses iniciales.

\section{USUFRUCTO Y PROYECCIÓN DE AUTORIDAD}

Un común denominador del crimen organizado es la capacidad que genera de investir de autoridad a sus ejecutores, en especial (pero no exclusivamente) a los capos y sus principales ayudantes. Esto es consecuencia de las prácticas de violencia, dominación y corrupción inmanentes al ejercicio criminal en entornos predeterminados. Así, consolidan en el imaginario colectivo de las estructuras sociales en las que se desenvuelven, no solo la idea, sino la manifestación efectiva de poder que trae aparejada la consolidación de la autoridad.

Esta deconstrucción del crimen se concentra entonces en la alta carga simbológica y estética que produce el mundo criminal. Los tatuajes, la vestimenta, las alhajas, los gustos musicales, los automóviles, los esquemas de seguridad personal y otros lujos son algunos de los elementos que ayudan a posicionar la imagen del criminal como un objeto, o bien, deseable, o bien, poderoso y siempre digno de temor o respeto. Esto es lo que Weber (1958) consideraba un tipo de autoridad carismática.

A esta serie de factores se suma un gran apalancador (tangencial si se quiere) de este estilo de vida: los medios de comunicación. Las necesidades comerciales del cine y la televisión han encontrado en la criminalidad un 
nicho de mercado casi inagotable que, además, está empezando a traspasar las consideraciones morales que obligaban a los criminales a fenecer o ser neutralizados, y pasan ahora a proyectarlos como agentes con mayor poder, fuerza, autoridad y control. En ese sentido, el crimen como entretenimiento ha cimentado su lugar en la cultura popular y ha hecho cada vez más difusa la frontera con la información (Dowler, Fleming y Stephen, 2006, p. 837).

Así, los noticieros, programas de crónicas, análisis e investigación periodística terminan cayendo en el dilema existente entre informar y servir de apología involuntaria a la criminalidad. En la mayoría de ocasiones, y pese a los códigos de ética y responsabilidad corporativa, los medios de comunicación son un elemento replicador del crimen y el terrorismo en la medida en que con la publicación de sus actividades muestran la efectividad y el éxito de los actos terroristas y los golpes de la criminalidad. En ese sentido, diseminan la viabilidad racional y operativa de las amenazas.

Por otra parte, la estética, la producción y la proyección social del crimen guardan además una relación con la necesidad de romper la lógica del anonimato inmanente a los procesos de urbanización y ampliación rural. La necesidad de destacarse y diferenciarse es otra de las consecuencias resultantes del hacer parte de cualquier subcultura y la criminal no es la excepción. De hecho, las estructuras criminales son una de las más exitosas en ese proceso en la medida en que las actividades que realizan ya son en sí mismas un comportamiento diferencial frente al resto de actividades de la sociedad, lo cual les da visibilidad y reconocimiento como amenaza para las autoridades y como modelo de ejemplo para otros criminales.

\section{LA NECESIDAD DE SUSCRIBIRSE A ESTRUCTURAS DE PROTECCIÓN EN ZONAS GRISES DE PRESENCIA ESTATAL}

Lo que Iazzetta y O'Donnell llaman zonas grises del Estado (Iazzeta, 2007), no son otra cosa que zonas geográficas y áreas de acción en la que este no tiene presencia y capacidad; o al menos en las que las tiene en forma muy reducida. Amenazas como el crimen organizado y el terrorismo usualmente han aprovechado esos vacíos geográficos e institucionales de poder para emplazar su autoridad y dominación (Stergios, 2001). Así, la gente que habita en zonas apartadas o que interviene en actividades desreguladas, usualmente se suscribe a las estructuras de autoridad ilegal que detentan los criminales y terroristas, quienes se vuelven entonces los proveedores de la seguridad, el equilibrio y la estabilidad que el Estado no alcanza a otorgar.

Ello tiene un componente adicional y es la efectividad ilegal en el control de actividades sociales. A diferencia del sistema legal regular que precisa de un aparataje institucional y un proceso burocrático para hacer efectiva la exigibilidad de los derechos, las estructuras criminales operan con una simplicidad más elevada que hace expeditas sus disposiciones y más efectivas sus acciones, lo cual permite la legitimación tácita de sus procesos por parte de la sociedad, sea a través de la confianza, o del temor y el constreñimiento. 


\section{EL INTERÉS DE GENERAR, ALIMENTAR, PRESERVAR O CONTRIBUIR A LA GENERACIÓN IDENTITARIA}

Otro de los productos sociológicos del crimen organizado es la generación de identidades. Si bien es cierto que esto puede estar perdiendo fuerza en la medida en que las estructuras criminales tienden a ser cada vez menos jerarquizadas y permanentes, existe sin embargo una generación identitaria que se suscribe a una serie de ideas, valores, códigos de comportamiento, lealtades y prescripciones que, de hecho, profundizan la creación de las subculturas criminales (Gobierno de Canadá, 2010) a las que ya se hizo referencia.

Al respecto, la teoría del aprendizaje ha hecho aproximaciones al contexto del crimen transnacional organizado. Aduce que la formación de identidades individuales responde a estímulos sociales. En ese sentido, resalta el contexto societal en el que se insertan y se generan identidades colectivas y no las individualidades (Crossman, 2012).

Esto resulta especialmente atractivo para agentes sociales con tendencias segregacionistas o antisociales como las pandillas juveniles; y en general para personas y grupos con deficiencias o aversiones estructurales frente a la construcción de identidades nacionales (Faget, 2013). Ello puede explicar por qué la construcción de nuevos proyectos nacionalistas e identitarios se soporta y, en ocasiones, se amalgama con la reproducción del crimen transnacional organizado como sucedió en la Serbia de Milosevic, Sudán del Sur, Rusia tras el colapso soviético o el fenómeno de las Maras en Centroamérica en pleno proceso de redemocratización de la década de los noventa.
Así, muchas de las estructuras criminales organizadas proveen sentido de pertenencia, búsqueda de objetivos predeterminados, bienestar económico, noción de colectividad dada a través de la internalización de un sentimiento de hermandad o comunidad a la que se suscriben, responsabilidad frente a la estructura, y un orden establecido de roles, acciones, tareas, poderes y relaciones de dominación (Lyman y Potter, 2007). Ejemplo de ello son tal vez las más tradicionales mafias internacionales y pandillas transnacionales: la camorra, la N'Dragheta en Italia, los VoryZakone en Rusia, los Klanes albaneses, la Yakuza japonesa, las Triadas chinas, los Yardíes jamaiquinos, las Maras en Centroamérica e incluso los Zetas y el cartel de Sinaloa en la misma región denotan patrones comunes en materia de generación de productos identitarios que, además, también incorporan toda una serie de elementos estéticos de los que ya se habló y que refuerzan la identidad del grupo criminal diferenciándolo del resto de la sociedad, haciéndolo respetable y temible.

\section{LA NECESIDAD DE GENERAR MECANISMOS} PARALELOS DE REGULACIÓN SOCIAL

Aparejado con la capacidad que tienen de brindar protección en zonas de alta y media desregulación geográfica e institucional, no son pocos los grupos criminales que van más allá y constituyen mecanismos de regulación social que, aun siendo tácitos, son más complejos y profundos. En efecto, el control social, visto desde la sociología, hace referencia a la capacidad que tiene una sociedad de regularse a sí misma (Janowitz, 1975). Las dinámicas 
ilegales no son menos efectivas por su condición desviada de la ley. Por el contrario, en ocasiones la operatividad ilegal para regular los comportamientos sociales es más eficiente, fluida y directa. La razón de ello estriba en la necesidad que tienen esas estructuras de trascender las etapas predatorias del crimen que obligan al uso de altos niveles de violencia, para situarse en nuevos estadios simbióticos e incluso parasitarios, considerados como los hitos de evolución natural de la criminalidad organizada en su proceso de consolidación.

En Sâo Paulo, Brasil, por ejemplo, el Primero Comando da Capital (PCC) es el grupo criminal más importante y grande de la ciudad. El nivel de consolidación que han alcanzado actualmente les ha permitido desarrollar mecanismos de regulación social como los llamados "Debates", que no son otra cosa que un juicio sumario en el que el líder de un barrio o cuadrante urbano toma decisiones punitivas sobre temas y personas que la misma sociedad pone a su consideración (Badrán, 2014). Anteriormente, los comportamientos solo se regulaban con el uso indiscriminado y ejemplarizante de la violencia. Hoy los debates amplían el rango de "penas alternativas" y la sociedad responde a esa mecánica de organización, bien por coerción o bien por convicción.

El caso colombiano también es ilustrativo, especialmente en zonas de la periferia territorial, en donde guerrillas y grupos paramilitares constrińeron, guiaron y predeterminaron la forma de la conducción y la acción social durante años. Este caso es aún más complejo porque originó estructuras completas de acción paraestatal en las que había producción ilegal de bienes y servicios, impuestos (extorsión), comercio internacional (tráfico), esquemas de seguridad, mecanismos de control y vigilancia, justicia sumaria, contabilidad y maquinaria electoral (cooptada); es decir, todos o casi todos los elementos de los que dispone un Estado regular para funcionar correctamente.

\section{LA NECESIDAD DE FINANCIAR CAUSAS CONSIDERADAS MORALMENTE SUPERIORES}

Con el constreńimiento por parte del sistema internacional a Estados que patrocinaban y financiaban el terrorismo, a sus ejecutores no les quedó más alternativa que plegarse de una manera más decidida e incremental hacia el crimen organizado como estrategia y fuente de financiación. En ese sentido, el crimen organizado empieza a tener alcances y puntos de hibridación con el terrorismo, que se erige usualmente revestido de una superioridad moral construida a través de hábiles estrategias discursivas de vocación religiosa o nacionalista.

La misma actividad criminal organizada entraña ciertos elementos morales, axiomas que justifican su materialización en función de determinantes históricos, deterministas, contextuales y coyunturales que hacen del crimen organizado un comportamiento fecundo, deseable y beneficioso para varios actores (van Duyne, Maljevic, Antonopulos, Harvey y von Lampe, 2015).

Ejemplo de ello es posible encontrarlo en la financiación de Hezbollah en la zona de triple frontera Brasil, Paraguay, Argentina, que le reporta a la organización cerca de 20 millones de dólares al año (Shelley, 2014). 
Del mismo modo, atentados terroristas emblemáticos como el 11 de septiembre, el 11 de Marzo, Beslán y Bali contaron con un fuerte componente logístico y financiero provisto por el crimen transnacional organizado.

Por tal motivo, la instrumentalización del crimen organizado para objetivos no económicos puede ser considerada efectivamente como otra de las razones que alimenta el utilitarismo sociológico del crimen organizado.

\section{EN SUMA...}

El crimen transnacional en su natural curso evolutivo va más allá de la trascendencia de etapas y estructuras criminales. Su transmutación empieza a tocar otras amenazas a la seguridad como sucede con el terrorismo y las guerras civiles. Sus escenarios geopolíticos cambian también hacia una concentración que combina las preferencias urbanas con las zonas desreguladas, áreas de conflicto, prisiones y fronteras. Por otra parte, la tendencia y los objetivos iniciales que dieron origen al fenómeno, amplían su espectro de acción. La necesidad del lucro económico viene aparejada con una necesidad creciente de regulación social, de construcciones simbólicas de poder y creaciones de subculturas identitarias que abren la puerta a un análisis del crimen organizado como objeto racionalmente deseable y elegible por parte de actores sociales más allá de los aspectos económicos tradicionalmente abordados.

Esta condición sociológica de la criminalidad no tiene realmente un distingo geográfico. La naturaleza sociológica del crimen organizado es la misma en casi todo el mun- do. Lo que cambia en el contexto geográfico es la representación en la que se manifiesta. La necesidad de generar valores identitarios, mecanismos de autoridad, regulación social y presencia paralela a los mecanismos institucionales de representación son comunes en todos los sistemas de crimen organizado, especialmente en aquellos de modelo padrino en donde la autoridad, la jerarquía y las funciones dentro del grupo se encuentran cada vez más definidas como sucede con las triadas chinas, las yakuzas japonesas, los zakone rusos, los clanes albaneses, la camorra y la N'drageta italianas, entre otros.

Esto da cuenta, entonces, de aquello que John Agnew (1998) resalta con razón: el hecho de que las fronteras y manifestaciones sociales ya no están contenidas en las fronteras geográficas. El crimen transnacional organizado como comportamiento social constructor de violencia, tiene un carácter cada vez más desterritorializado, su naturaleza comporta comunes denominadores que no distinguen raza o nacionalidad porque se ubican en patrones lógicos de acción humana más básicos e instintivos: supervivencia, poder y dominación.

\section{¿UTILITARISMO SOCIOLÓGICO EN EL CRIMEN TRANSNACIONAL QUE ATRAVIESA COLOMBIA? EL EJEMPLO DEL TRÁFICO DE MIGRANTES}

Para nadie es un secreto que el país suramericano es un nodo central en una amplia gama de actividades donde el crimen se complementa eficientemente con el aspecto transnacional (McDermott, 2014). En esto se combinan la 
histórica falta de presencia del Estado sobre las zonas de frontera y sus limitaciones al momento de brindar servicios públicos a la población, con la tradición de ilegalidad que reviste la historia reciente de Colombia, sobre todo en lo que se refiere al narcotráfico. También hay que tener en cuenta la localización geográfica del país, que enclavado en una zona de transición entre el norte y el sur del continente americano, es nodo central en la consolidación de rutas de múltiples tráficos ilegales transnacionales. Esta condición territorial termina por generar también otros tipos de incentivos para la perpetuación del crimen transnacional.

Una de las actividades criminales transnacionales que más ha ganado terreno en Colombia en el último par de años es el tráfico de migrantes (UnODC, 2013; Daugherty, 2015; Palma, 2016). Ante la competencia en torno al tráfico de drogas, de armas, de fauna, de bienes de consumo y de minerales -por solo mencionar algunos-, la opción de canalizar el paso de personas que de manera irregular buscan atravesar la geografía del país se ha consolidado como un negocio rentable, económica y sociológicamente hablando. No obstante, antes de hablar de las posibilidades que esta actividad plantea en términos de utilitarismo sociológico cabe hacer algunas precisiones al respecto.

\section{TRATA Y TRÁFICO DE PERSONAS}

Para hablar de tráfico de migrantes hay que hacer una diferencia conceptual con la trata de personas. Por ser un fenómeno relativamente reciente en Colombia, lo primero suele con- fundirse con lo segundo. De hecho, la línea que los separa es tan delgada que a veces parece inexistente.

Por regla general, la trata se refiere al desplazamiento involuntario de individuos con fines de trabajo forzoso o de esclavitud. En otras palabras, la trata implica la coacción o falta de voluntad del individuo que se ve involucrado como bien traficado (Lee, 2007; Shelley, 2010). Colombia ha sido escenario de este fenómeno, como exportador de personadas tratadas durante varias décadas.

Los casos más sonados son los de mujeres, niñas y niños víctimas tratados con fines de explotación sexual. Pero ningún tipo de personas está exento de ser víctima de este delito. Hoy hay mercado para trabajos forzosos en campos que van desde la minería o la agricultura hasta el empleo en sweatshops (talleres clandestinos) y maquilas alrededor del mundo (Hillstrom, 2013). Más recientemente se han identificado casos de personas tratadas para el trabajo en construcción en regiones como el Golfo Pérsico, y se han reportado crecientemente casos de bebés y niños tratados con fines de comercialización y venta en el mercado negro internacional (Childs, 2016).

Por su parte, el tráfico es una actividad criminal cuyos ejecutores actúan como agentes de paso para personas que tienen la intención de desplazarse a través de las fronteras nacionales, pero que en la gran mayoría de los casos, no cuentan con los permisos para hacerlo (Sánchez, 2014).

Estas personas pueden ser calificadas como migrantes irregulares que, por su misma condición, para atravesar las fronteras de los Estados por los que transitan o que tienen 
como destino requieren de los servicios de los traficantes. Entre las funciones de estos últimos se encuentran administrar las rutas de paso fuera del control institucional, proveer los servicios de transporte, de protección y de alojamiento, entre otros, dentro de una amplia gama de acciones que conforman la cadena de valor de esta actividad. De cualquier forma, los migrantes suelen recurrir a los traficantes en la creencia -materialmente sustentada o no- de que su gestión disminuye los riesgos y quizás los costos en los que incurrirían si emprendieran el viaje por sí solos.

Mediante el seguimiento a varios grupos de traficantes, en Colombia se han logrado establecer algunos indicadores que dan cuenta del fenómeno.

\section{AlgunOS DATOS QUE ILUSTRAN LA REALIDAD COLOMBIANA}

Según Migración Colombia, en 2014 fueron encontrados más de 2.100 migrantes irregulares en diferentes redadas contra bandas de traficantes. En 2015 la cifra creció más de cuatro veces. Según la misma entidad, habrían sido 8.855 personas las reportadas (El Tiempo, 2016). A partir de cálculos preliminares, en 2016 ya se habría sobrepasado este número.

Aunque estas cifras son apenas la punta del iceberg - por la naturaleza clandestina de este delito-, ellas ilustran su tendencia al aumento. Según el Wall Street Journal (2015), Panamá (siguiente punto de tránsito para la mayoría de migrantes irregulares) reportó en 2014 la llegada de más de 8.000 personas desde Colombia. Para el primer semestre de
2015 este número habría superado los 10.000 y en 2016 la cifra parece en aumento.

Varios factores explican esta situación. Uno de ellos es la apertura de rutas de migración irregular -ante la saturación de algunas de las más antiguas, como la transpacífica y la caribeña- que ponen a Colombia como punto de paso obligado de miles de personas que buscan llegar a Estados Unidos.

El caso cubano es quizá el más notorio. Desde 2012, el Gobierno de Cuba comenzó un proceso de liberalización en materia migratoria, lo cual implica que muchos de sus ciudadanos puedan salir y entrar de la isla con cierta autonomía. Este hecho acabó por coincidir con la liberalización de la política de visados de países como Ecuador y la suscripción de acuerdos entre ambos gobiernos, lo que ha traído como resultado el aumento de los flujos migratorios de cubanos. Ante esta situación, el Gobierno ecuatoriano decidió restablecer los visados de turismo para los ciudadanos cubanos a partir de noviembre de 2015 (ANDES, 2015). Las presiones de los vecinos como Colombia, pero también de gobiernos centroamericanos directamente afectados incidieron en esto. Nicaragua, desde mediados de 2015, y más recientemente Costa Rica y Panamá han declarado su intención de cerrar el tránsito de cubanos irregulares por su territorio (Meléndez, 2015).

Por citar un ejemplo, según el Gobierno colombiano, a 2015 en la frontera colomboecuatoriana confluían 76 pasos ilegales que hacen del país un paso obligatorio (Diario del Sur, 2015). Esto se replica en la zona del Darién, que termina conectando a los mi- 
grantes con destinos como Panamá y Estados Unidos. Personas provenientes del Levante, de Asia sudeste, de África e incluso de Haití, estarían utilizando esta ruta. Son ya más de un centenar los casos reportados de ciudadanos de Bangladesh o de Somalia, así como varias decenas de personas procedentes de Ghana, Nepal y Mali (Martínez, 2015).

La crisis fronteriza entre Colombia y Venezuela de 2015 puso en evidencia la extensión de este fenómeno en esta zona. Entre la crítica situación generada a partir de la deportación de centenares de colombianos desde el vecino país, se reportaron casos de personas que, provenientes de Cuba, África subsahariana e incluso el subcontinente indio, estarían atascadas en un limbo jurídico propiciado por el cierre de la frontera entre los dos países (Cosoy, 2015).

Por otra parte, el cerramiento del paso por parte de las autoridades panameńas durante el primer semestre de 2016 ha acentuado la presencia de migrantes irregulares en toda la zona del Urabá colombiano, volviendo esta área un caldo de cultivo para que traficantes colombianos y de otras nacionalidades se lleven una lucrativa tajada del negocio (Onzaga, por publicar).

Los puntos de mayor tránsito de migrantes irregulares serían los terminales terrestres de Ipiales, Pasto y Medellín, el puerto de Turbo en Antioquia -el principal nodo del tráfico de migrantes desde Colombia hacia Panamá-, Apartadó, el río Cacarica y Puente América -ya en el tapón del Darién-. Muchos de los migrantes llegarían a Suramérica por Brasil y pasarían a Colombia vía Perú, y luego Ecuador (Serna, 2015).
Esto conlleva una situación humanitaria tremendamente tensa. A agosto de 2015 se habían reportado sendos casos de extorsión, violación y crímenes derivados a extranjeros que se encontraban atravesando el país ( $S_{e-}$ mana, 2015). En 2016, las denuncias de los migrantes continuaban. Muchos de ellos no solamente estaban siendo violentados por las varias redes de traficantes existentes en el país, sino por actores irregulares "tradicionales" como las guerrillas y estructuras de crimen organizado; así mismo, se presentaron indicios de abusos varios por parte de la Policía Nacional (ElTiempo, 2015; Onzaga, por publicar).

\section{RASGOS DEL TRÁFICO DE MIGRANTES en Colombia}

Los rasgos peculiares del tráfico de migrantes y las especificidades del caso colombiano dificultan su seguimiento y control por parte de las autoridades en por lo menos tres aspectos específicos.

La primera dificultad resulta del espacio donde tiene lugar el tráfico y de la naturaleza de su objeto: los individuos.

Como la gran mayoría de los tráficos ilícitos, el de migrantes se vale de las zonas donde la presencia del Estado es limitada o tiende a ser disputada. Los epicentros del tráfico son las sensibles zonas fronterizas - como Nariño y Putumayo con Ecuador; y el Urabá antioqueño y chocoano con Panamá- donde la ausencia del gobierno se asocia con la porosidad de las fronteras.

A lo anterior se suma la disposición de los individuos traficados de colaborar (hasta cierto punto) con sus traficantes, con el objeto 
de llegar a su destino. Esta posibilidad por supuesto no existe cuando se habla de tráfico de bienes inertes.

La segunda complicación surge de la oportunidad de negocio que representa la migración irregular para las organizaciones criminales existentes. El campo de acción criminal en materia de tráfico de migrantes ha estado prácticamente libre y solo desde hace poco comenzó a ser tenido en cuenta por las autoridades.

Hay que tener en cuenta que el tráfico de migrantes se vale también de las rutas existentes para otro tipo de movimientos ilícitos. Se producen así economías de escala para los traficantes. Los migrantes pueden servir como correos humanos de drogas u otros bienes ilícitos o extraídos ilegalmente.

Hay indicios muy serios al respecto. En el Urabá antioqueño y chocoano se ha reportado una suerte de cohabitación en torno a los retornos del negocio entre grupos armados organizados, como el hoy denominado Cartel del Golfo y las Farc (Prieto, 2014). Estos grupos dominarían el tráfico en Turbo, el puerto y su zona de influencia sobre el litoral, mientras que aquellos tendrían el control del negocio sobre la parte continental, en selva próxima al tapón del Darién. Ambos grupos estarían traficando migrantes y otros bienes ilícitos a través de las mismas rutas y medios.

La tercera dificultad proviene de la naturaleza transnacional del problema. De aquí la necesidad de una respuesta conjunta entre el Gobierno colombiano y los gobiernos vecinos, que incluya el plano municipal y no apenas central. Estas respuestas, además, tendrían que incluir lo político, lo policial y lo humanitario.
Lo anterior implica intercambios de información entre la fuerza pública de los países afectados, el seguimiento conjunto de las rutas migratorias existentes, y comenzar a pensar en centros de recepción y acogida en los puntos de mayor concentración de migrantes.

\section{TRÁFICO DE MIGRANTES Y UTILITARISMO SOCIOLÓGICO DEL CRIMEN TRANSNACIONAL EN COLOMBIA: HIPÓTESIS PARA UNA AGENDA DE ESTUDIO}

Esta rápida radiografía permite comenzar a pensar en la existencia de rasgos que soportarían en la práctica la existencia de múltiples rasgos de utilitarismo sociológico en el caso del tráfico de migrantes en Colombia. A continuación, se postulan cinco hipótesis específicas de acuerdo con los criterios expuestos en la primera parte de este texto.

\section{Proyección de autoridad y perpetuación de prestigio}

El tráfico de migrantes en Colombia, según la poca información empírica existente hasta el momento, se vale de estructuras (físicas, organizacionales y sociales) previamente existentes, centrales en otros tipos de flujos irregulares que atraviesan las fronteras del país. La generación de nuevos réditos y nuevas oportunidades de negocio, en este caso, a través de la consolidación de estructuras puntuales de tráfico de personas, se constituiría en mecanismos de generación de autoridad y de perpetuación de prestigio para tres grupos de traficantes.

Por una parte, es útil para aquellos que con experiencia previa en estructuras crimi- 
nales tienen la posibilidad de administrar o generar una nueva fuente de ingreso a su estructura. Por otra parte, este nuevo nicho de negocio constituye una opción de movilidad jerárquica para mandos bajos y medios dentro de la estructura criminal, a través de la especialización y gestión del nuevo nicho de mercado. Por último, la novedad de la actividad constituye una oportunidad de inserción dentro del prestigioso mundo criminal para terceros actores, locales, que quieran entrar al tráfico, abriéndose así un espacio dentro del amplio mercado ilegal de frontera.

El asunto de fondo es que el tráfico no solo es una oportunidad económica frente a una oferta de personas dispuestas a atravesar la geografía colombiana, sino que se constituye en una nueva línea de negocio, en la que emprendedores del tráfico pueden obtener también réditos en términos de prestigio y autoridad al interior de las estructuras criminales existentes. Esto, por supuesto, no es exclusivo de un fenómeno como el del tráfico de personas, pero en el caso en cuestión se convierte en una lógica dentro del utilitarismo sociológico de esta actividad, en tanto esta es una actividad novedosa dentro del contexto analizado.

\section{Tráfico de personas como mecanismo de suscripción a estructuras de protección en medio del desgobierno}

En este punto es interesante pensar en la demanda por traficantes y redes de tráfico. Las personas que buscan atravesar la geografía nacional de manera irregular con el fin de llegar a un tercer destino, muchas veces tie- nen escasos o nulos conocimientos sobre las lógicas institucionales que se presentan en el terreno que atraviesan. Además, por su condición irregular, no tienen la posibilidad de acercarse a la estructura institucional oficial, salvo arriesgando su posibilidad de éxito en el trayecto. De esta forma, el traficante y las personas que administran servicios alrededor del tráfico son los únicos que están en capacidad de interceder o interactuar, de alguna forma, por las personas traficadas.

Se genera así una aleación entre las instituciones de gestión y promoción del tráfico al margen de la ley como estructuras de protección de los migrantes. De hecho, estas serían el único recurso, nexo e interlocutor de los traficados con el orden social por el que atraviesan. Esto termina generando mayor capacidad de maniobra de los traficantes frente a la "mercancía”, pero también afirma su rol dentro del espectro socioeconómico analizado, en tanto la demanda por tráfico siga existiendo.

Esta característica es particularmente interesante en el caso de este tráfico porque, en últimas, al ser personas y no mercancías inertes, estas se encuentran en capacidad de reproducir la información a otros prospectivos migrantes, especificando así sobre los roles que juegan los empresarios del tráfico, ratificando su autoridad, su prestigio, pero también convirtiéndose en el referente de protección en medio del proceso. Aunque, por supuesto, no hay aún suficiente evidencia empírica que sostenga esta premisa, cabe pensar en la posibilidad de que esto apoye la generación de réditos económicos, pero también, termine por posicionar la actividad 
criminal como algo deseado, sobre todo en el espectro de desgobierno que implica afianzarse en la zona de frontera.

\section{Identidad del traficante frente}

\section{a los migrantes: otredad y etnocentrismo}

Sin duda, el componente de otredad que implica la interacción entre migrantes provenientes de tantos y tan diversos orígenes, se convierte en un punto de análisis si se tiene en cuenta el asunto de la creación de identidad como forma de utilitarismo sociológico. La capacidad de liderazgo derivada de la forma misma que toma la interacción entre migrantes y traficantes - por la fuerza, la coerción, la necesidad, o por la razón que sea-, puede tener una caracterización peculiar si se tienen en cuenta diferencias evidentes como nacionalidad de los migrantes, etnia (que para este caso funcionaría como raza) o idioma.

Encarnado dentro de la óptica de la etnificación de las actividades económicas -lo cual incluye las actividades criminales-, el tráfico de migrantes se convierte en una oportunidad para que sus ejecutores entren en contacto con personas que cuentan con evidentes rasgos diferenciadores (Light y Gold, 2000). Por la naturaleza y posición dentro de la ruta de tráfico, estas diferencias se convierten en elementos que acentúan la posición del ejecutor de la actividad dentro del tráfico. No es lo mismo hablar del traficante en general, que del traficante colombiano que conoce la zona, cuenta con un nexo frente a las instituciones locales y además lidera en su zona de influencia; igualmente, no es lo mismo ejercer dominio frente a un migrante cubano que habla el idioma y se presume culturalmente cercano, que frente a una persona proveniente de Pakistán, Siria o Eritrea.

Este componente, aunque puede ser tildado de determinista culturalmente hablando, se sostiene sobre posiciones sociológicas básicas como las de Levi-Strauss (1999), que señalan que el etnocentrismo es una fuerza inherente al comportamiento humano cuando entra en contacto con personas provenientes de composiciones culturales diferentes a la propia. Esto es también posible en una lógica social que entrańa una actividad económica ilegal -como el tráfico de migrantes- que implica a la larga las interacciones entre individuos provenientes de diferentes órdenes sociales. Esta categorización de otredad y etnocentrismo puede terminar por refirmar la identidad del traficante, generando así nuevos incentivos para el utilitarismo sociológico del crimen.

\section{Mecanismos paralelos de regulación social}

La proliferación de estructuras dedicadas al tráfico de migrantes en Colombia ha sido evidente conforme la demanda por estas se ha acentuado. Como el resto de tráficos que se gestan en y a través del país, se han ido estableciendo diferentes mecanismos que regulan las interacciones entre diferentes grupos. Para el caso en cuestión, es interesante observar dos fenómenos frente al tráfico de migrantes. Por una parte, la cohabitación entre estructuras enemigas - del tipo guerrillas y Grupos Armados Organizados- en torno a los roles que toman dentro del tráfico. Por otra parte, es también relevante tener en cuenta la distribu- 
ción geográfica que el control sobre el tráfico de migrantes ha venido tomando.

Heredado de las economías de escala que se han generado a través de la cohabitación criminal de estructuras guerrilleras con bandas criminales en torno al negocio del narcotráfico ${ }^{1}$, el tráfico de migrantes parece apuntar hacia la misma dirección. Las bandas criminales concentran su acción en los nodos de paso de los migrantes, como en Turbo (Antioquia), y reciben réditos de las transacciones por transporte, alojamiento y otros similares durante el paso de las personas. Las estructuras guerrilleras que se encuentran presentes sobre el terreno de la frontera con Panamá se hacen cargo del paso de la frontera, además de cargar a los migrantes con otras mercancías.

Esto inserta los dos aspectos mencionados en términos de eficiencia y sostenibilidad del tráfico. Hay una especialización en las acciones, al tiempo que hay una distribución de réditos según la especialidad. Esto genera una economía de escala, solo posible frente a los acuerdos tácitos encontrados entre las estructuras involucradas, que termina por regular socialmente el asunto en las zonas donde se encuentran presentes. Cabe pensar que mientras estos acuerdos se respeten, terceros agentes asociados al tráfico de manera voluntaria o involuntaria, tácita o explícita se pueden ver beneficiados a través de la prestación de terce- ros servicios, como alojamiento, alimentación y otros similares. Esto en últimas constituye otro incentivo desde el nivel sociológico para que el tráfico se perpetúe.

\section{El tráfico de migrantes como parte de una causa moralmente superior}

En el caso del tráfico de migrantes, no solamente en Colombia, sino alrededor del mundo, el imperativo moral de supervivencia es utilizado -de manera legítima o ilegítimapor las personas que recurren a este tipo de servicios al margen de la ley. Para muchos, recurrir al tráfico de personas es el resultado de la inexistencia de mecanismos legales que apoyen su voluntad de supervivencia.

Piénsese en el caso de una persona que ha salido de su país a causa de un conflicto violento que pone en serio riesgo su supervivencia o la de su familia, y en la que el Estado de su procedencia no tiene o bien la capacidad o bien la voluntad de garantizarle este derecho básico. Aunque puede acercarse a estructuras legales existentes como el derecho de asilo, lo cierto es que, en medio de las diferentes crisis internacionales, los sistemas que contemplan vías regulares para acceder a este derecho se han saturado. Mientras tanto, el riesgo aumenta. Así, las opciones de supervivencia comienzan a rayar con la ilegalidad. El tráfico

1 En el caso de Tumaco (Nariño), durante mucho tiempo las Farc han dominado las grandes rutas de tráfico de estupefacientes en las zonas rurales, mientras que las bandas criminales se han hecho con el microtráfico. Los beneficios de mantener este statu quo son mayores que los costes en los que se incurriría al entrar en disputa por el dominio absoluto de ambas esferas (Palma, 2014). 
es un mecanismo que termina apoyando la intención de supervivencia de los individuos involucrados.

El traficante puede justificar su acción, entonces, en términos de la provisión de un servicio que en últimas cuenta con una intención moralmente enclavada dentro de la perspectiva del apoyo a la supervivencia a las personas que buscan sus servicios. Algunos de los argumentos más sonados al momento de la captura de los presuntos implicados en estas actividades son del tipo "solo estamos prestando un servicio" o "estamos salvándoles la vida”. En esto hay una dualidad que raya entre la legalidad de la acción y una lectura peculiar específica sobre el propósito de la acción criminal. De cualquier forma, esto ilustra otra posibilidad de utilitarismo sociológico encarnada en el crimen transnacional.

\section{CONCLUSIÓN}

El utilitarismo sociológico se manifiesta de diferentes formas en el crimen transnacional. Varios elementos, que se salen del análisis tradicional del fenómeno, fundados en el racionalismo económico, pueden ser expuestos a través de esta perspectiva. Este texto propuso una agenda de análisis con cinco elementos concretos por observar: el usufructo y la proyección de la autoridad; la necesidad de suscribirse a estructuras de protección en zonas grises de presencia estatal; el interés de generar, alimentar, preservar o contribuir a la generación identitaria; la necesidad de generar mecanismos paralelos de regulación social, y la necesidad de financiar causas consideradas moralmente superiores.
La aplicación de este modelo frente a la evolución del incipiente fenómeno del tráfico de migrantes en Colombia se convierte en una primera oportunidad para comenzar a pensar en el tema, de cara al entendimiento de otros fenómenos similares. No obstante, queda en el tintero el desarrollo ulterior de estos conceptos a través de evidencia concreta. Para esto sería necesario desarrollar nuevos estudios el respecto, que incluyan indagaciones in situ. Así, esto se convierte en una excusa para seguir haciendo indagaciones al respecto.

\section{REFERENCIAS}

Agencia Pública de Noticias del Ecuador y Suramérica - ANDES (2015). Ecuador solicitará visa a cubanos y reitera exhorto a EE.UU. a que suspenda politicas migratorias hacia Cuba. Recuperado de http://www.andes.info.ec/es/noticias/ecuadorsolicitara-visado-cubanos-reitera-exhorto-eeuususpenda-politicas-migratorias-hacia

Agnew J. (1998). Geopolitica: una re-visión de la politica mundial. Estados Unidos: Trivilus.

Albini, J. y McIlliwain, J. (2012). Deconstructing organized crime: A Historical and Theorical Study. London: McFarland.

Badrán, F. (2014). L'evolution du crime transnational organisé en Amerique du Sud: le cas du colombie, bresil et venezuela. Paris: IHEAL Paris III.

Berdal, M. y Serrano, M. (eds.) (2002). Transnational Organized Crime and International Security. Business as Usual? Boulder: Lynne Rienner

Childs, A. (2016). Why child trafficking surges after natural disasters. CNN Freedom Project. Recuperado de http://edition.cnn.com/2016/03/23/ opinions/child-trafficking-natural-disasters/ 
Cosoy, N. (2015). El limbo que viven en Colombia los médicos cubanos que desertaron en Venezuela y quieren llegar a Ee.uU. ввC Mundo. Recuperado de http://www.bbc.com/mundo/ noticias/2015/08/150824_colombia_medicos_ cubanos_protesta_ep

Crossman, A. (2012). Social Learning Theory. Estados Unidos: About Education.

Daugherty, A. (2015). Ola de tráfico humano llega a Colombia. InsightCrime. Recuperado de http:// es.insightcrime.org/analisis/ola-trafico-humanollega-colombia

Diario del Sur (2015). Sueńo americano se trunca en Ipiales. Recuperado de http://diariodelsur.com. $\mathrm{co} /$ noticias/nacional/sue $\% \mathrm{C} 3 \% \mathrm{~B} 1$ o-americanose-trunca-en-ipiales-129631

Dowler, K., Fleming, T. y Stephen, M. (2006). Constructing Crime: Media, crime and popular culture. Canadian Journal of Criminology and Criminal Justice, 837-865.

El Tiempo (2015). Colombiano en tres dias, la trampa de redes de tráfico de migrantes. Recuperado de http://www.eltiempo.com/politica/justicia/ trafico-de-migrantes-la-trampa-de-las-redes-quese-dedican-a-este-delito/16392632

El Tiempo (2016). Cifra de migrantes ilegales se cuadruplicó en 2015. Recuperado de http://www. eltiempo.com/politica/justicia/entrevista-conchristian-kruger-sarmiento-director-de-migracion-colombia/16478459

Faget, J. (2013). Sociologie de la délinquance et de la justice pénale. Toulouse: Érès.

Gobierno de Canadá (2010). Identity-Related Crime: A Threat Assessment. Canadá: Minister of Public Safety of Canadá.

Hillstrom, C. (2013). Escape from an L.A. Sweatshop: How modern-day slaves become lobbyists. Yes! Magazine. Recuperado de http://www.yesmagazi- ne.org/issues/the-human-cost-of-stuff/breakingfree-from-an-american-sweatshop

Iazzeta, O. (2007). Democracias en busca de Estados. Rosario: Homosapiens.

Janowitz, M. (1975). Sociological Theory and Social Control. American Journal of Sociology, 81 (1), 82-108.

Lee, M. (ed.) (2007). Human Trafficking. Portland: Willand Publishing.

Levi-Strauss, C. (1999). Raza y cultura. Madrid: Altaya.

Light, I. y Gold, S. (2000). Ethnic Economies. Sommerville: Emerld Group Publishing.

Lyman, M. y Potter, G. (2007). Organized Crime. Estados Unidos: Prentice Hall.

Martínez Arango, R. (2015). Preservar la vida de migrantes, misión de la Armada en Urabá. El Colombiano. Recuperado de http://www.elcolombiano. com/antioquia/crece-el-trafico-de-migranteshacia-eu-por-uraba-armada-MJ2865691

McDermott, J. (2014). Perspectivas 9. Friederich Ebert Stiftung en Colombia. Recuperado de http://library.fes.de/pdf-files/bueros/la-seguridad/11053. pdf

Meléndez, J. (2015). Costa Rica cierra sus fronteras al paso de cubanos. El Nuevo Herald. Recuperado de http:/www.elnuevoherald.com/noticias/mundo/ america-latina/cuba-es/article44572392.html

Onzaga, T. (por publicar). Tráfico de migrantes a través de Colombia. Los límites del régimen internacional (2005-2016) [Trabajo de grado para optar por el título de Magíster en Relaciones Internacionales]. Bogotá: Pontificia Universidad Javeriana.

Palma, M. (2014). Tumaco: ¿Razones para la desesperanza? Bogotá: Instituto de Ciencia Política / Fedepalma.

Palma, M. (2016). Ascenso, proliferación, gestión y ¿control? Tráfico de migrantes a través de las fronteras de Colombia. En Molano-Rojas, A. Fronteras en Colombia como zonas estratégicas: 
análisis y perspectivas. Bogotá: Instituto de Ciencia Política y Konrad Adenauer Stiftung.

Paoli, L. (ed.) (2014). The Oxford Handbook of Organized Crime. New York: Oxford University Press.

Prieto, C. (2014). La relación FARC-Bacrim y sus lugares comunes. fIP Opina. Recuperado de http://www. ideaspaz.org/publications/posts/1068

Rabasa, A., Boraz, S., Chalk, P., Cragin, K., Karasik, T., Moroney, J., O’Brien, K. y Peters, J. (2007). Ungoverned Territories: Understanding and Reducing Terrorism Risks. Santa Monica: Rand Corporation.

Rahimi, S. y Graumans, R. (2015). Reconsidering the Relationship between Integration and Radicalization. Journal for Deradicalization, 15/16 (5).

Rivelois, J. (2004). Collusions, Connivences et Actions Criminelles. Paris: IRD.

Sánchez, G. (2014). Human Smuggling and Border Crossings. London: Routledge.

Schneiders, T. (2014). Salafismus in Deutschland. Ursprünge und Gefahreneinerislamisch-fundamentalistischen Bewegung. Bielefeld: Transcript.

Semana (2015). El infierno perdido de los migrantes. Recuperado de http://www.semana.com/nacion/multimedia/el-infierno-perdido-de-losmigrantes/426329-3

Serna Osorio, J. (2015). Migrantes, bomba de tiempo que amenaza a Turbo. Recuperado de http:// www.elcolombiano.com/antioquia/seguridad/ migrantes-bomba-de-tiempo-que-amenaza-aturbo-XB1892231
Shelley, L. (1995). Transnational organized crime: an imminent threat to the nation-state? Journal of International Affairs, 48 (2), 463-489.

Shelley, L. (2010). Human trafficking: A global perspective. Cambridge: University Press.

Shelley, L. (2014). Dirty entanglements: Corruption, crime, and terrorism. Virginia: George Manson University.

Stergios, S. (2001). Economics of Governance. Springer Verlag, 173-202.

unODC (2013). Dimensión del delito de tráfico de migrantes en Colombia. Realidades institucionales, legales y judiciales. Bogotá: UNODC.

van Duyne, P., Maljevic, A., Antonopulos, G., Harvey, J. y von Lampe, K. (2015). The relativity of wrongdoing: Corruption, organised crime, fraud and money laundering in perspective. The Netherlands: Wolf Legal Publihers.

Vertovec, S. (1999). Conceiving and researching transnationalism. Ethnic and Racial Studies, 22 (2), 1-25.

Vidino, L. y Brandon, J. (2012). Countering Radicalization in Europe. ICSR, King's College Policy Report. London: King's College.

Wall Street Journal (2015). Panama's Perilious Jungle is a New Route for Migrants. Recuperado de http://www.wsj.com/video/panama-perilousjungle-is-a-new-route-for-migrants/6EFF3A4ED45D-492D-B16C-EC065D672A06.html

Weber, M. (1958). The three types of legitimate rule. Society and Institutions, 4 (1): 1-11. 